( (NASA-CR-199740) ROSAT HRI OBSERVATIONS OF MAGELLANIC CLOUD SUPERNDVA REMNANTS

(Harvard-Smithsonian Center for Astrophysics) $10 \mathrm{p}$
N96-15188

Unclas

G3/89 0081112 


\title{
ROSAT HRI OBSERVATIONS OF MAGELLANIC CLOUD SUPERNOVA REMNANTS
}

\author{
John P. Hughes \\ Harvard-Smithsonian Center for Astrophysics \\ Email ID \\ jph@cfa.harvard.edu
}

\begin{abstract}
Analysis of deep ROSAT high resolution imager (HRI) observations of two oxygen-rich supernova remnants (SNRs) in the Magellanic Clouds is described. For N132D, I exploit the limited spectral information provided by the HRI to investigate arcsecond scale spectral variations. I find that there is a region of harder X-ray emission near the southern limb and regions of softer emission near the center and northwestern limb. The remnant is believed to be interacting with a molecular cloud and the harder emission to the south is explained as a result of increased absorption along the line-of-sight there. I argue that the softer emission comes from X-ray emitting material with an enhanced abundance of oxygen. For the second SNR, E0102.2-72.2, the spatial structure is investigated in detail using two-dimensional image fitting techniques. Evidence is found for a ring-like and a spherically symmetric shell-like component both of which were modeled as homogeneous regions. In addition, a significant fraction of the observed flux $(\sim 11 \%)$ must come from a resolved clumped component. A comparison with optical and radio imagery is made to provide a physical basis for the components identified in the X-ray analysis. The mass of X-ray emitting gas in the remnant is estimated and a value of $\sim 75 M_{\odot}$ was determined. The dominant uncertainty on this quantity is the extent of unresolved clumping in the X-ray gas. Such clumping would tend to reduce the mass estimate by $f^{1 / 2}$, where $f$ is the mean volume filling factor of the gas.
\end{abstract}

\section{Introduction}

N132D and E0102.2-72.2 are the brightest supernova remnants (SNRs) in soft X-rays in each of the Large and Small Magellanic Clouds (LMC and SMC), respectively. They belong to the class of oxygen-rich remnants, which include such Galactic examples as Cassiopeia A and G292.0+1.8. SNRs in this class show optical spectra with strong red- and blue-shifted oxygen emission lines indicating matter velocities of several $1000 \mathrm{~km} \mathrm{~s}^{-1}$. Since $\mathrm{H} \alpha$ is usually not detected from the high velocity filaments, it is likely that they are metal-rich fragments of the stellar ejecta from the core collapse of a massive star. Studies of the stellar ejecta in these remnants are important to our understanding of the evolution of massive stars and the associated nucleosynthesis which occurs in these systems.

\section{N132D}

The high velocity oxygen-rich filaments in N132D appear to be distributed in a ring-like morphology with a diameter of $\sim 6 \mathrm{pc}$ (Lasker 1980) and expansion 


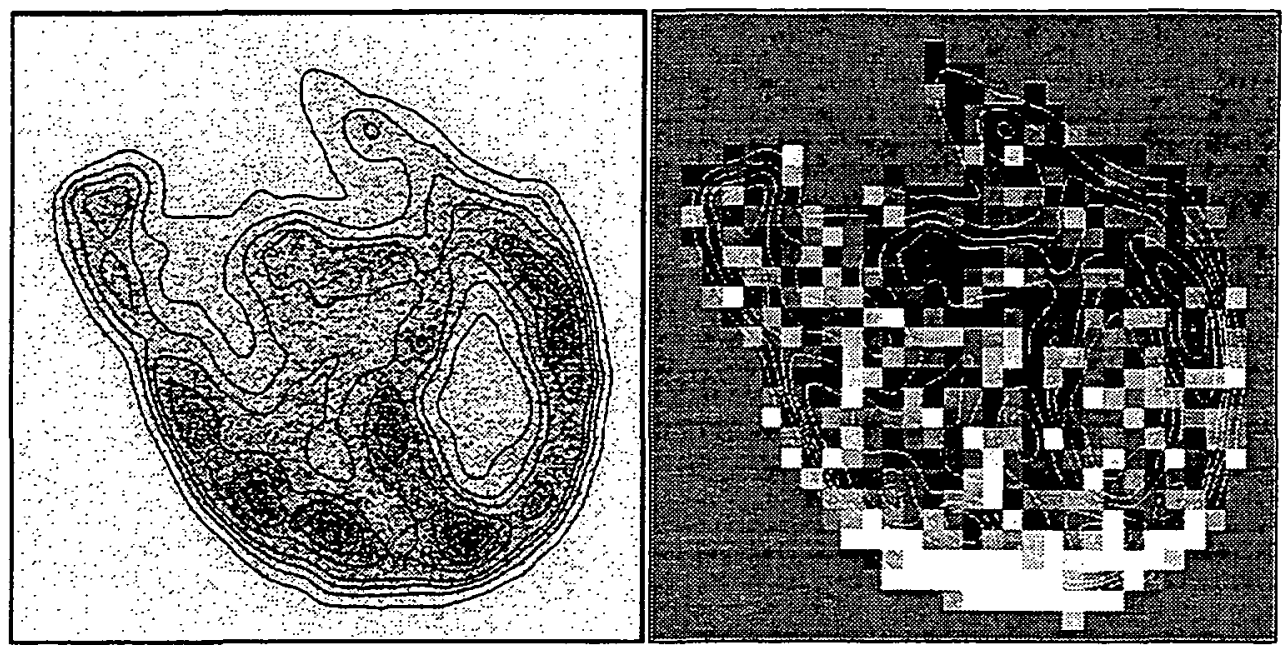

FIG. 1.-(Left) ROSAT HRI image of N132D where the grayscale presents the detected events, and the contours show the data after two iterations of the Lucy-Richardson deconvolution algorithm. Contours are shown at values of $0.5,1,1.5,2,3,4,5$, and 6 counts s arcmin $^{-2}$. (Right) ROSAT HRI pulse height centroid significance map displayed from values of -3 to 3 in units of $\sigma$. Each panel is $128^{\prime \prime}$ square, which corresponds to a linear size of $31 \mathrm{pc}$ assuming a distance of $50 \mathrm{kpc}$ to the LMC.

velocities $\left(\sim 2250 \mathrm{~km} \mathrm{~s}^{-1}\right)$ implying an age of 1300 yr assuming undecelerated motion. Surrounding these filaments is a relatively quiescent limb-brightened shell of emission with a diameter of about $21 \mathrm{pc}$ (for a distance to the LMC of $50 \mathrm{kpc}$ ). The inferred oxygen abundance is high in the central filaments, 10-100 times the abundance in the outer shell. A dual morphology is also seen in the $\mathrm{X}$-rays. Analysis of the $\mathrm{X}$-ray surface brightness variations in the outer shell suggested that the supernova explosion occurred in a low density cavity in the interstellar medium formed by the $\mathrm{H}$ II region of the precursor star (Hughes 1987). The most recent $\mathrm{X}$-ray analysis of N132D was the nonequilibrium ionization (NEI) modeling of spectral data from the Einstein Observatory reported by Hwang et al. (1993) (hereafter referred to as HHCM), in which it was concluded that, for the entire remnant, oxygen was overabundant relative to iron by a factor of at least 1.9 compared to the solar ratio. I utilize the results from this work in my interpretation of the spectral information from the ROSAT HRI.

N132D was observed by the ROSAT HRI for some $2.7 \times 10^{4} \mathrm{~s}$ in 1991 February. Within a 100" radius of its center there were 123911 total counts of which about 1450 were background events for a source counting rate of $4.564 \pm$ $0.013 \mathrm{~s}^{-1}$. Fig. 1 (left-hand panel) shows the ROSAT HRI image of N132D. The two spatial components, a limb-brightened clumpy shell, as well as excess emission near the center, are evident.

\subsection{ROSAT Pulse Height Analysis}

The ROSAT HRI has 15 pulse height (PH) channels with the potential to provide some limited energy resolution. Calibration data taken at the MPE Panter Calibration Test Facility before launch show that the centroid of the HRI PH distribution increases from 3.3 to about 5.4 as the energy of the incident $\mathrm{X}$-rays varies from 0.183 to $1.49 \mathrm{keV}$ (see Table 6 in David et al. 1993). For this observation of $\mathrm{N} 132 \mathrm{D}$, the PH centroid, averaged over the whole SNR, was $4.5578 \pm 0.0054$. 
Maps of the PH centroid and its statistical error were made for N132D using $4^{\prime \prime}$ square pixels. Next a significance map was determined by subtracting the mean centroid value for the entire remnant from the $\mathrm{PH}$ centroid in each pixel and then dividing by the statistical error on the centroid in each pixel. The significance values ranged between plus and minus $4 \sigma$ (for the 499 image pixels with counts between 68 and 676). If there were no intrinsic spatial variations in the PH centroid across the remnant, then, by construction, the distribution of significance values should be gaussian with mean $\mu=0$ and width $\sigma=1$. In fact a $\chi^{2}$ test showed that this distribution could be rejected at a confidence level $\gg 99.95 \%$. The observed distribution was considerably broader, $\sigma=1.30$, strongly suggesting that the distribution of $\mathrm{PH}$ centroid values shows some intrinsic variation with position. Since the data were taken in "wobble" mode, spatial variations of the HRI gain should be averaged over, although this point needs additional study. I note for completeness that there was no correlation between the counts in a given pixel and the significance value there.

The PH centroid significance map is shown in Fig. 1 (right-hand panel). Only pixels containing 50 counts or more are plotted. The most dramatic feature of this image is the obvious structure which appears in it. Most of the high significance values (implying higher mean photon energies) lie together in the southern region of the remnant, while most of the low significance values (lower energies) lie in two regions: one near the center and another near the northwestern limb of the remnant. I determined the average $\mathrm{PH}$ centroid by summing the data from these various regions and found the following values: northwestern $4.404 \pm 0.015$, central $4.363 \pm 0.022$, southern $4.708 \pm 0.015$. These values differ from the mean for the entire remnant by about 10 times the statistical error.

\subsection{Discussion}

By interpolating the calibration data, I can relate an observed $\mathrm{PH}$ centroid value to a mean detected photon energy. For the softer regions (northwestern and central) the HRI PH centroid implies a mean photon energy of $0.83 \mathrm{keV}$, for the harder region (southern) the implied mean photon energy is $1.00 \mathrm{keV}$, and for the remnant as a whole, $0.92 \mathrm{keV}$. The latter value can be compared to the mean photon energy predicted from the best-fit NEI model of HHCM. Including the efficiency of the HRI and the effective area of the X-ray telescope, I obtain a predicted value for the mean photon energy of $0.92 \mathrm{keV}$. Although this excellent agreement in predicted versus observed mean photon energy for the entire remnant is very encouraging, until considerably more attention is paid to the known temporal variations of the HRI gain, the agreement should be considered largely fortuitous. On the other hand, the observed spatial variations in $\mathrm{PH}$ centroid values across N132D will be considerably less sensitive to temporal gain variations and thus the conclusions drawn below should be reliable.

A few years ago, strong $\mathrm{CO}$ emission from a molecular cloud was discovered just south of N132D and it was suggested that there was an interaction between the SNR and the cloud (Hughes, Bronfman, \& Nyman 1991). Fig. 2 shows the map of molecular emission superposed on the Einstein HRI image. These data provide a natural explanation for the observed hardening of the X-ray spectrum toward the south: I propose that there is increased X-ray absorption along the line-of-sight there. I can reproduce the observed mean photon energy of 1.00 $\mathrm{keV}$ in this region if I increase the column density in the HHCM NEI model (leaving all other parameters fixed) to a value of $\sim 2.5 \times 10^{21} \mathrm{~cm}^{-2}$. This is a factor of 4 more than the best-fit value of column derived for the entire remnant. As the explanation for the central region of softer X-ray emission, I propose 


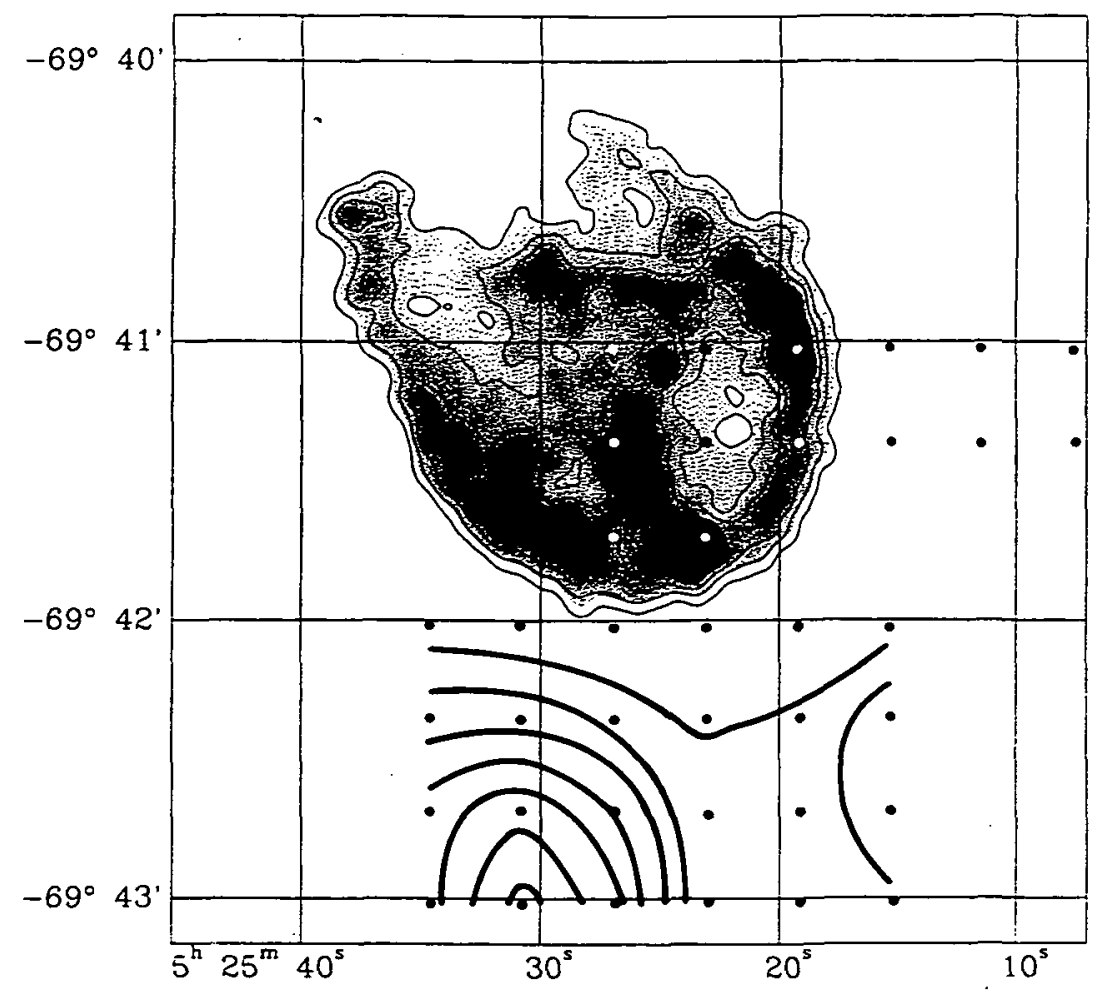

FIG. 2.- Einstein HRI image of N132D overlaid with contours of velocity-integrated CO emission. The grid pattern for the $C O$ observations is shown. The contour levels are $3,5,7,9,11$, 13 , and $15 \mathrm{~K} \mathrm{~km} \mathrm{~s}^{-1}$ for a velocity integration range from $260 \mathrm{~km} \mathrm{~s}^{-1}$ to $270 \mathrm{~km} \mathrm{~s}^{-1}$.

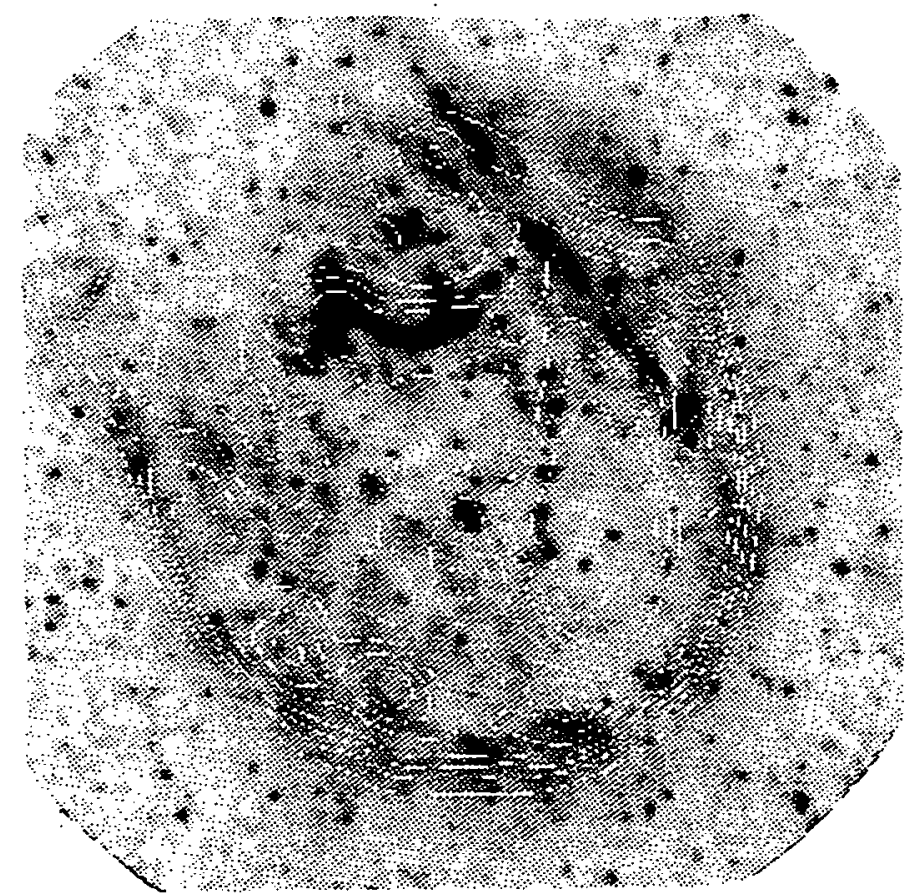

FIG. 3.- Optical image of N132D in the light of [O III] $\lambda$ 5007. This map shows only the slow-moving ( $v \lessgtr 150 \mathrm{~km} \mathrm{~s}^{-1}$ ) material. 
enhanced oxygen emission. First, I point out that the mean photon energy for an X-ray emitting plasma composed of pure oxygen would be $0.76 \mathrm{keV}$, assuming the same spectral parameters (i.e., column density, temperature, ionization state) as the HHCM best-fit NEI model. Thus it is possible to explain the observed mean photon energy of $0.83 \mathrm{keV}$ from the central regions of $\mathrm{N} 132 \mathrm{D}$ by enhancing the oxygen abundance there by a factor of 10 or more (once again keeping the other model parameters fixed). Given the good positional agreement between the softer X-ray emission and the known oxygen-rich optical filaments, $I$ believe that it is reasonable to draw the conclusion that oxygen is enhanced in the central X-ray emitting regions of N132D.

An optical image of N132D showing the slow-moving material is given in Fig. 3 (J. Morse 1993, private communication). The bright linear feature in the northwest apparently has a soft counterpart in the PH significance map (Fig. 1). Since this feature appears fairly bright in $\mathrm{H} \alpha$ emission, it is unlikely that its brightness in [O III] $\lambda 5007$ and the softness of its X-ray spectrum are a result of oxygen enhancement. This might suggest that excitation or NEI effects are influencing its observational properties. Additional analysis may aid in understanding this curious feature, but it is likely that the poor spectral capabilities of the HRI will ultimately limit what we can learn.

This work has demonstrated the capabilities of the ROSAT HRI for providing spectral information on arcsecond spatial scales. With high enough signalto-noise it is possible to observe statistically significant spectral variations with position across extended sources. Interpretation of these variations in terms of interesting physical source quantities requires detailed knowledge of the average X-ray spectrum of the object and is (probably) restricted to constraining a single quantity at a time. Nevertheless, this capability, limited though it is, is currently unique and further effort should be devoted to its calibration.

\section{E0102.2-72.2}

E0102.2-72.2 was discovered as a strong soft X-ray source during a survey of the SMC by the Einstein Observatory (Seward \& Mitchell 1981). Its identification as an oxygen-rich SNR was made by Dopita, Tuohy, \& Mathewson (1981) and a velocity map of the remnant was presented by Tuohy \& Dopita (1983). These data showed that the oxygen-rich material covers a velocity range of $-2500 \mathrm{~km} \mathrm{~s}^{-1}$ to $+4000 \mathrm{~km} \mathrm{~s}^{-1}$. An Einstein HRI observation of the remnant (Inoue, Koyama, \& Tanaka 1983) showed a strongly limb-brightened shell of emission with a radius of about $20^{\prime \prime}$ ( $\sim 6 \mathrm{pc}$ assuming a distance to the SMC of $57.5 \mathrm{kpc}$ ). Analysis of these data indicated that the geometry of the X-ray emitting region of the remnant was more consistent with being ring-like than shell-like (Hughes 1988). Recently the US/Japanese satellite ASCA obtained an X-ray spectrum of the remnant which showed strong $\mathrm{K} \alpha$ line emission from $\mathrm{O}, \mathrm{Ne}$, and $\mathrm{Mg}$ with evidence for weaker emission from $\mathrm{Si}, \mathrm{S}$, and $\mathrm{Fe}$ (Hayashi et al. 1994). A high resolution radio map (Amy \& Ball 1993) shows a strongly limb-brightened shell with an angular diameter of $\sim 40^{\prime \prime}$. There is evidence from this image for a relatively strong compact radio component near the center of E0102.2-72.2.

The ROSAT HRI observed E0102.2-72.2 for $2.0 \times 10^{4} \mathrm{~s}$ in 1991 November. Within a radius of $40^{\prime \prime}$ there were 19500 total counts of which about 460 were background events for a source counting rate of $0.958 \pm 0.007 \mathrm{~s}^{-1}$. An image of the remnant, after two iterations of the Lucy-Richardson deconvolu- 

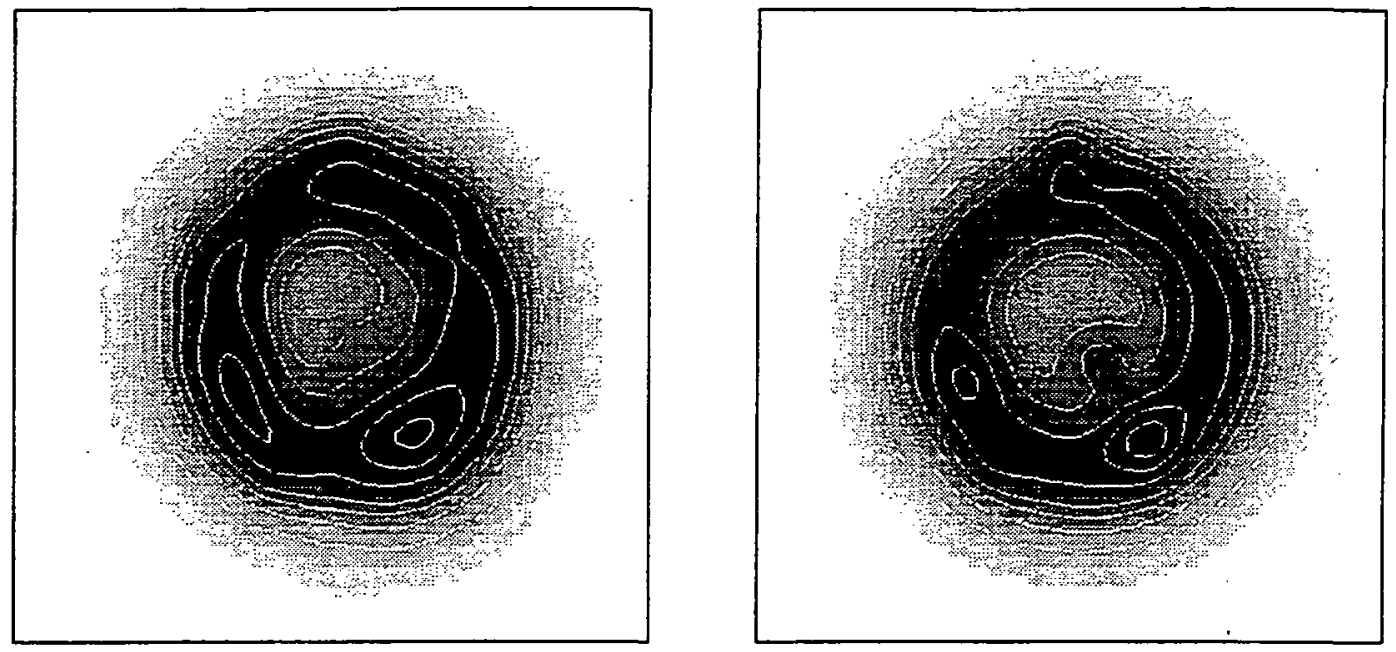

FIG. 4.-(Left) Deconvolved ROSAT HRI image of E0102.2-72.2. Contours are shown at values of $0.5,1,1.5,2,3,4$, and 5 counts $\mathrm{s}^{-1} \mathrm{arcmin}^{-2}$. (Right) The best-fit model for E0102.2-72.2 including both ring-like and shell-like components, plus six individual spherical clumps. The contour levels correspond to the same values as in the deconvolved data. Each panel is $64^{\prime \prime}$ square, which corresponds to a linear size of $18 \mathrm{pc}$ assuming a distance of $57.5 \mathrm{kpc}$ to the SMC.

tion algorithm, is shown in Fig. 4 (left-hand panel). A strongly limb-brightened, apparently clumpy, shell is evident.

\subsection{Spatial Model Fits}

I performed spatial fits to the two-dimensional $\mathrm{X}$-ray image in order to investigate the intrinsic geometric structure of the remnant. The fits were done using software specifically developed for the analysis of sparsely filled images. It employs a maximum likelihood statistical estimator derived for Poisson distributed data and parameter estimation is done using the downhill simplex method. Numerous possible geometric models, such as spherical shells, rings, spherical clumps, and so on, can be projected onto the sky and convolved with the ROSAT HRI point spread function for comparison to the data.

For ring models, I assumed that the plane of the ring was the same as the plane of the sky. The geometric parameters included position on the sky, the inner and outer radii of the ring, the opening angle of the ring, and a normalization. As can be seen in Fig. 4, the surface brightness of the ring appears to vary azimuthally from an average of 5.25 counts $\mathrm{s}^{-1} \operatorname{arcmin}^{-2}$ in the southwest to 2.88 counts s arcmin $^{-1}$ in the northeast. In order to model this brightness variation I allowed for a relative azimuthal variation in the mean plasma density in the ring using $n(\theta)=\left[1+\cos \left(\theta-\theta_{0}\right)\right] / 2+n_{0}\left[1-\cos \left(\theta-\theta_{0}\right)\right] / 2$. The relative density would vary smoothly, according to this function, from a maximum of 1 at the azimuthal reference angle, $\theta_{0}$, to a minimum of $n_{0}$ at angle, $\theta_{0}+180^{\circ}$. The surface brightness then scales as the square of the relative density times the geometric line-of-sight through the ring. (In the final model results presented below, the best-fit minimum density was $\sim 0.8$.) In total, eight parameters were needed to fully specify this type of component. Spherical shells were parameterized in an identical manner, with the ring opening angle fixed at the value needed to produce a complete shell $\left(90^{\circ}\right)$. Finally, clumps were modeled as constant density spherical regions; each required four parameters: position on the sky, clump radius, and a normalization. 
Initially, single component models were tried. Neither pure shell nor ring models provided decent fits. In the former case, the surface brightness in parts of the central $\sim 6^{\prime \prime}-8^{\prime \prime}$ radial region was overpredicted (by factors of 1.6) while in the latter it was significantly underpredicted (by up to factors of 3 ). For any single model fit our maximum-likelihood estimator did not yield an absolute goodnessof-fit criterion (although relative differences in the likelihood function between model fits can be accurately assigned confidence levels of relative probability, a feature I utilize below). To estimate the absolute goodness of any single best fit I compared radial averages of the model and data in quadrants and calculated $\chi^{2}$, taking care only to include radial bins with 25 or more counts in the data. For the single shell fits I obtained $\chi^{2}=718.5$ for $\nu=151$ degrees of freedom. For the pure ring models the best fit yielded $\chi^{2}=796.4$ for $\nu=150$.

It is not surprising that these simple models were such poor descriptions of the data. However, what was unexpected was the significant improvement in the quality of the fit obtained when a model with both a ring-like and a shell-like component was used. For simplicity I assumed the two components were concentric and showed the same azimuthal density variation. In this case, then, relative to the pure ring model, only three additional parameters: the radius, thickness, and normalization of the shell-like component, were required for a complete specification of the model. When the radially-averaged data and model were compared, the best-fit two-component model yielded $\chi^{2}=287.2$ for $\nu=147$, a tremendous improvement over the single component models. Nevertheless, for both observational and theoretical reasons, it is not expected that such a smooth, homogeneous, and unclumped model should accurately describe the structure of SNRs. Indeed, an examination of the difference image made by subtracting the data and this model revealed features on spatial scales of $\lesssim 5^{\prime \prime}$ with peak surface brightnesses approaching $\sim 1$ counts s$^{-1}$ arcmin $^{-2}$.

These features were modeled as discrete clumps as described above. Clumps were introduced into the model in an iterative fashion, by examining the difference image after each successive best-fit minimization and picking out new features by eye. The process was terminated when no obvious features could be discerned in the difference image. In total, six individual clumps were fit. The maximum-likelihood estimator decreased by a value of 265.5 when the 24 parameters of the clumped component were included. Since the difference of maximum-likelihood estimators is distributed like $\chi^{2}$ (for degrees of freedom equal to the number of new parameters), this result is highly significant. The fitted clumps range in diametric sizes from $2.9^{\prime \prime}$ up to $4.5^{\prime \prime}$. These are at the resolution limit of the HRI and therefore should probably be considered only upper limits to the true clump sizes. An image of the final two-component model including the clump component is shown in Fig. 4 (right-hand panel). Comparison with the data image shows peak surface brightness residuals at the level of $\sim 0.6$ counts $\mathrm{s}^{-1} \operatorname{arcmin}^{-2}$. Fig. 5 (left-hand side) shows the radially averaged data and model including a breakdown of the model into its various components. On the right-hand side of Fig. 5, residuals are shown for the four quadrants of the image. In general, as these various representations show, the agreement between the data and model is quite good.

\subsection{Discussion}

Detailed analysis of the ROSAT HRI X-ray image of E0102.2-72.2 shows evidence for both a ring-like component and a spherically-symmetric shell component. Other young oxygen-rich SNRs in the Galaxy, such as Cassiopeia A 

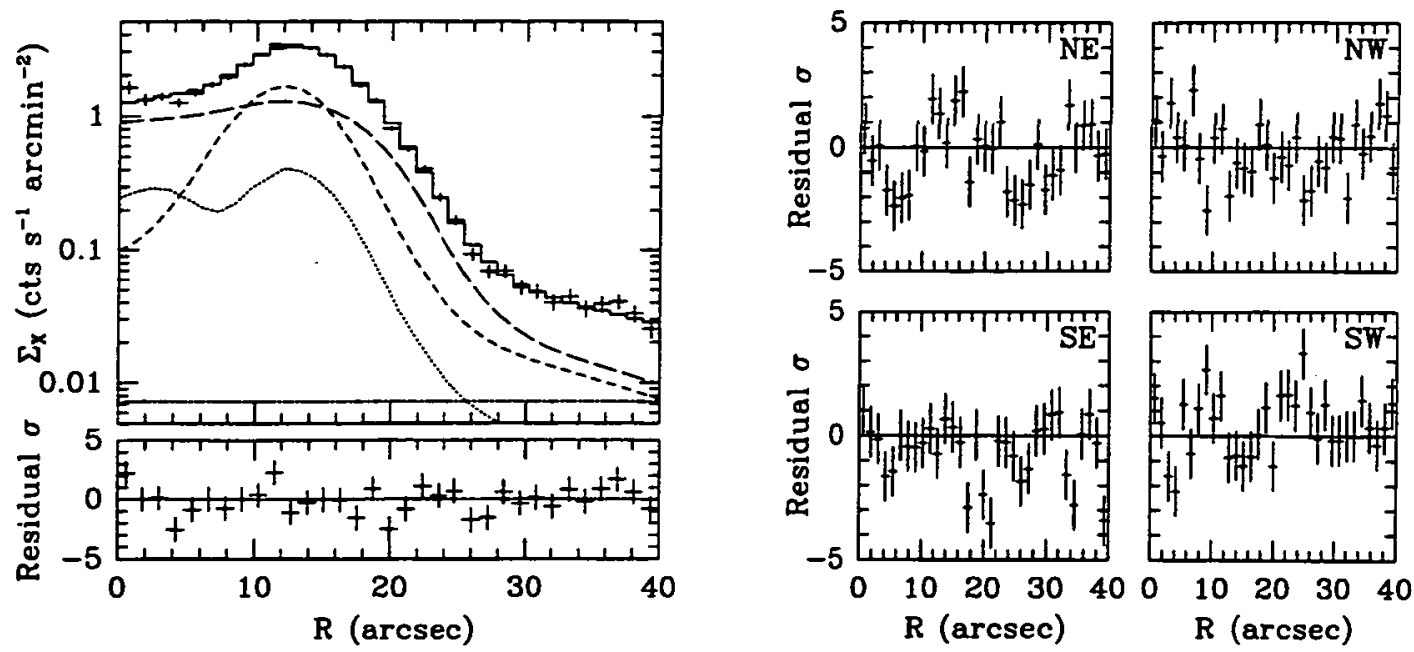

Fig. 5.-(Left) (Top) Radially-averaged X-ray surface brightness of E0102.2-72.2 compared to the best-fit spatial model. The several components to the model are shown separately. The short-dash curve is the ring-like component, the long-dash curve is the spherical shell component, the dotted curve is the clumped component, and the background is the straight line near the bottom of the panel. (Bottom) The difference between the observed data and the best-fit model in units of the statistical error in each radial bin. (Right) The difference between the observed data and the best-fit model for the four quadrants of the image as indicated.

and G292.0+1.8, have also been suggested to show such a dual morphological structure. For E0102.2-72.2 roughly 39\% of the HRI emissivity comes from the ring, $50 \%$ from the spherical shell, and $\sim 11 \%$ from a clumped component. Given the limited statistics and the instrumental spatial resolution, the latter is merely a lower limit to the clumped component.

Fig. 6 (left-hand panel) shows a schematic cross-sectional view of the bestfit model for the X-ray emission. It is interesting to note that the inner edges of both the ring and shell components were determined (by the fitting procedure itself) to be nearly equal. Most of the clumps appear to be associated with the ring-like component. It is plausible to identify the ring component with the ejecta and its evident clumpiness is consistent with the onset of the wellknown instabilities (Rayleigh-Taylor, Kelvin-Helmholtz) which tend to disrupt and fragment SN ejecta. The spherical shell (or some part of it) would arise from the swept-up circumstellar medium. This hypothesis would suggest that the integrated X-ray spectrum of the remnant should show evidence for two spectral components: one with low abundances as appropriate to gas in the SMC ( $\sim 20 \%$ cosmic) and another with enhanced abundances as from nucleosynthesis in a massive star (see mass estimate below). The $A S C A$ spectrum of E0102.2-72.2 provides some qualitative support for this picture (Hayashi et al. 1994).

Comparison with optical imagery provides further insight into the physical basis for the components identified in the X-ray analysis. In Fig. 6 (right-hand panel), I show the radially averaged surface brightness profile of E0102.2-72.2 in the light of [O III] $\lambda 5007$ from data taken at the CTIO 4-m in 1986 November. The stellar component was subtracted from this profile using a continuum band image, centered at $6100 \AA$, of the same region of the sky. The optical emission shows a local peak at the inner edge of the X-ray ring component (12.3") and then a gradual decrease as it is traced further out. By the time the outer edge of the ring is reached (17.2"), the optical emission has dropped to a minimum value. I believe that we are seeing cool, dense clumps of oxygen-rich ejecta en- 

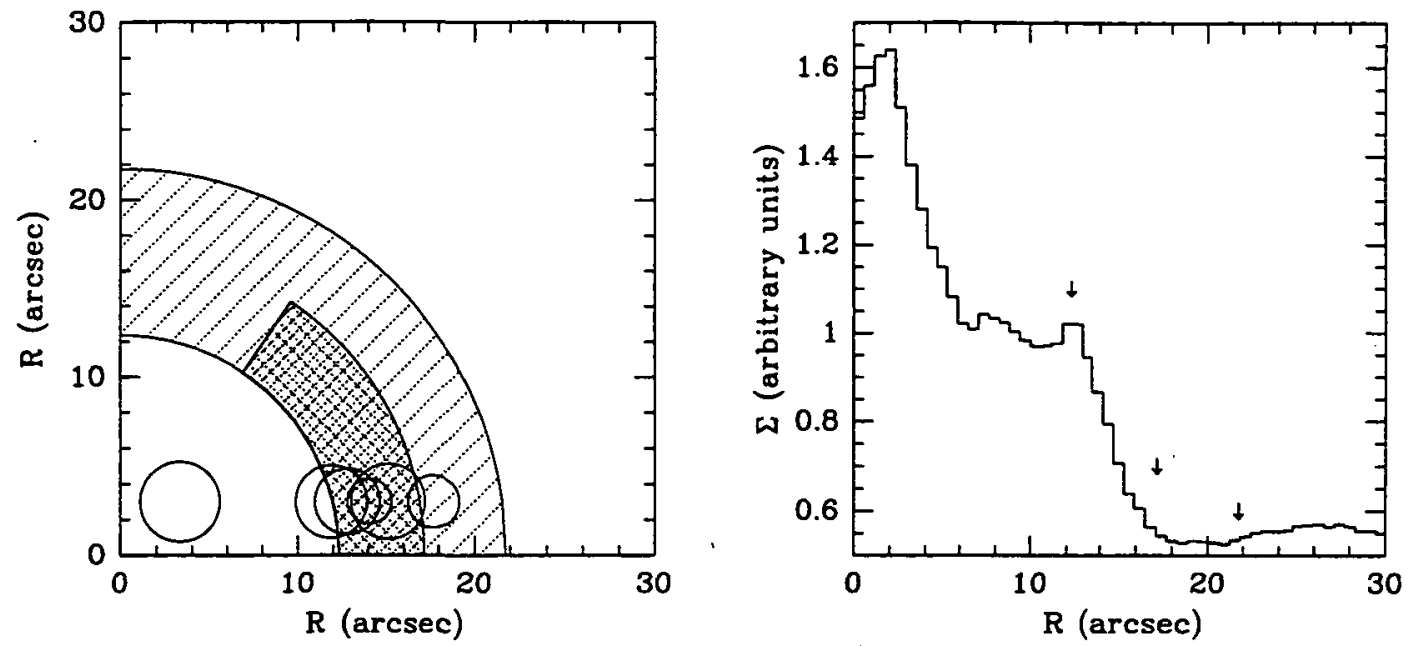

FIG. 6.-(Left) Cross-sectional view of the geometric model which best fits the ROSAT HRI image. The plane of the sky lies along the horizontal axis and runs in and out of the page. The viewing direction lies along the vertical axis. The ring component is shown as the doubly hatched region, while the spherical shell component is shown singly hatched. The radial positions of the six fitted clumps are drawn to scale as circles near the bottom of the figure. (Right) Radiallyaveraged surface brightness of E0102.2-72.2 in the light of [O III] $\lambda 5007$ after subtraction of the stellar component. The arrows denote, in increasing order from the center, the location of the inner edge of the ring component, the outer edge of the ring, and the outer edge of the spherical component.

tering the reverse shock and then gradually being destroyed in the high pressure environment of the hot X-ray emitting ejecta.

Beyond the blast wave (as indicated by the outermost extent of the Xray emission $Z 22^{\prime \prime}$ ), there is an optical halo, presumably a fossil $\mathrm{H}$ II region photoionized by the precursor star, the UV flash of the SN, or the UV radiation from the SNR itself (Dopita et al. 1981). I confirm that this halo is indeed suppressed in the $\mathrm{X}$-ray emitting region extending from the outer edge of the ring component to the location of the blast wave itself, as first suggested by Dopita et al. (1981).

The correlation between the $\mathrm{X}$-ray and radio data is not particularly good in detail. The radio shell appears to be somewhat asymmetric, with its outer diameter varying around the remnant azimuthally by some $\pm 5^{\prime \prime}$. This level of asymmetry is just not present in the X-ray data. Furthermore the brightest part of the radio shell is toward the northeast, where the X-ray shell shows its minimum brightness. There is, however, a weak X-ray clump near the position of the relatively strong, compact central component found in the radio map which Amy \& Ball (1993) speculate might be a plerionic component. This would be an exciting discovery, since, if true, it would signal the presence of a pulsar in the remnant. The X-ray clump contains only about $1.7 \%$ of the total SNR flux for an HRI counting rate of $\sim 0.016 \mathrm{~s}^{-1}$. The only known oxygen-rich shell remnant with a plerionic core component is the LMC SNR E0540-69.3, which has a $R O S A T$ HRI rate of $0.187^{-1}$ (Seward \& Harnden 1994). After correcting for the difference in distances to the two remnants, the inferred X-ray intensity for the putative plerionic clump in E0102.2-72.2 is about a factor of 10 less than the intensity of the known plerion in E0540-69.3. Using the empirical relationship between the spin-down energy loss rate for a pulsar, $\dot{E}$, and the X- 
ray luminosity of its associated plerion (Seward \& Wang 1988) I estimate $\dot{E}$ to be $\sim 10^{37.5} \mathrm{erg} \mathrm{s}^{-1}$ for the putative pulsar in E0102.2-72.2. The period can also be estimated; a value of $\sim 0.14 \mathrm{~s}$ is indicated, assuming an age of $1000 \mathrm{yr}$ and the $\dot{E}$ given above. Although these values are fully consistent with those observed for other known young pulsars, it is still quite possible that the compact central component is merely a bright spot in the SNR shell seen projected onto the center of the remnant. A definitive result requires the detection of pulsations (this is difficult in the X-ray band because of the limited number of detected events) or the measurement of a flat radio spectrum from the core component.

Finally I turn to an X-ray-derived estimate for the mass of the SNR. In addition to the geometric model for the structure of the emission region, this estimate requires a spectral model to relate the observed count rates to emission measure. For this I use results from fits of a NEI model (see Hughes \& Singh 1994) to the $A S C A$ data for E0102.2-72.2 (Hayashi et al. 1994). I estimate the densities in the remnant to be $n_{S} \sim 3.7 \mathrm{~cm}^{-3}$ for the shell component and $n_{R} \sim 6.0 \mathrm{~cm}^{-3}$ for the ring component (after accounting for the physical overlap of the geometric models). For simplicity I ignore the contribution of the fitted clumps to the estimate. This leads to a total mass of

$$
M \sim\left(15 f_{R}^{1 / 2}+60 f_{S}^{1 / 2}\right) D_{57.5 \mathrm{kpc}}^{5 / 2} M_{\odot} .
$$

Note the strong dependence of the derived mass on distance. This demonstrates the power of studying SNRs in the Magellanic Clouds where the uncertainty in distance introduces less than a $25 \%$ error in the derived mass. The result also explicitly includes the dependence on small scale clumping of the gas (i.e., at spatial scales below the instrumental resolution), parameterized by the volume filling factors, $f_{R}$ and $f_{S}$. Even for the rather extreme value of 0.1 , the progenitor of E0102.2-72.2 clearly was a massive star.

\section{References}

Amy, S. W., \& Ball, L. 1993, ApJ, 411, 761

David, L. P., Harnden, F. R., Jr., Kearns, K. E., \& Zombeck, M, V, 1993, The ROSAT High Resolution Imager, U.S. ROSAT Science Data Center report

Dopita, M. A., Tuohy, I. R., \& Mathewson, D. S. 1981, ApJ, 248, L105

Hayashi, I., Koyama, K., Ozaki, M., Miyata, E., Tsunemi, H., Hughes, J. P., \& Petre, R. 1994, PASJ, to appear June 1994

Hughes, J. P. 1987, ApJ, 314, 103

Hughes, J. P. 1988, in IAU Colloquium 101, The Interaction of Supernova Remnants With the Interstellar Medium, 125

Bughes, J. P., Bronfman, L., \& Nyman, L. 1991, in Supernovae, The Tenth Santa Cruz Summer Workshop in Astronomy and Astrophysics, 679

Hughes, J. P., \& Singh, K. P. 1994, ApJ, 422, 126

Hwang, U., Hughes, J. P., Canizares, C. R., \& Markert, T. H. 1993, ApJ, 414, 219 (HHCM)

Inoue, H., Koyama, K., \& Tanaka, Y. 1983, in IAU Symposium 101, Supernova Remnants and Their X-Ray Emission, 535

Seward, F. D., \& Harnden, F. R. 1994, ApJ, in press

Seward, F. D., \& Mitchell, M. 1981, ApJ, 243, 736

Seward, F. D., \& Wang, Z. R.. 1988, ApJ, 332, 199

Tuohy, I. R., \& Dopita, M. A. 1983, ApJ, 268, L11

This research was supported in part by NASA Grant NAG5-1724 and Smithsonian Institution funds. 